\title{
Permanent Tracheostomy in a Thoroughbred Mare with Arytenoid Chondritis
}

\section{Soomin Park ${ }^{1}$ \\ Kyung-won Park ${ }^{1}$ \\ Eun-bee Lee' \\ Yongwoo Sohn ${ }^{2}$ \\ Hyohoon Jeong ${ }^{1}$ \\ Tae-Young Kang' \\ Jong-pil Seo,*}

${ }^{1}$ College of Veterinary Medicine and Veterinary Medical Research Institute, Jeju National University, Jeju 63243, Korea

${ }^{2}$ KRA Veterinary Center, Korea Racing Authority, Gwacheon 13822, Korea
Abstract A 10-year-old Thoroughbred mare was referred to the Jeju National University Equine Hospital with roaring, dyspnea, and weight loss. On endoscopic examination, the horse was diagnosed with right arytenoid chondritis. Surgical treatment was selected due to the failure of a previous medical treatment. Permanent tracheostomy was performed in a standing position. The horse was restrained and tied in a proper position in the stock. The cranial parts of the 2 nd to 5 th tracheal cartilages were resected, as were the associated skin, mucosa, muscle, and cartilages. After the stoma was formed, external mucosa and skin were sutured using a simple interrupted method. The horse was hospitalized for 22 days receiving postoperative care including antibiotics, non-steroidal anti-inflammatory drugs, dressing as required, and was pregnant six months after the surgery. A permanent tracheostomy is thought to be effective in horses with diseases causing upper respiratory tract obstruction.

Key words permanent tracheostomy, arytenoid chondritis, upper respiratory tract obstruction, horse.

*Correspondence: jpseo@jejunu.ac.kr

\section{ORCID}

Soomin Park:

https://orcid.org/0000-0002-1116-1091

Kyung-won Park:

https://orcid.org/0000-0002-6637-2208

Eun-bee Lee:

https://orcid.org/0000-0003-3654-5654

Yongwoo Sohn:

https://orcid.org/0000-0002-3732-5322

Hyohoon Jeong:

https://orcid.org/0000-0002-9646-9991

Tae-Young Kang:

https://orcid.org/0000-0002-2591-9713

Jong-pil Seo:

https://orcid.org/0000-0002-6418-9813

Copyright $\odot$ The Korean Society of Veterinary Clinics 


\section{Introduction}

Arytenoid chondritis is an inflammation within the laminar portion of the arytenoid cartilage. Arytenoid chondritis is characterized by a mass around the larynx, an area that is space-limited, resulting in roaring and exercise intolerance $(1,11)$. Arytenoid chondritis is common in young racehorses, and there is a higher incidence of left-sided chondritis than right-sided chondritis $(1,2,6)$. The disease can be treated both medically and surgically. In an early stage or emergent case, antibiotics and anti-inflammatory drugs can be administered as medical treatment (4). If the patient has not been treated for a long term, surgical treatment may be selected $(1,4,5)$. Surgical treatment options include arytenoidectomy and tracheostomy. Tracheostomy can be performed on a permanent or temporary basis $(1,5)$.

Permanent tracheostomy is a procedure creating a stoma in the anterior part of the trachea. This surgery is used to correct irreversible damage of upper respiratory organs that has resulted in permanent obstruction. Tracheal malformations, arytenoid chondritis, nasopharyngeal cicatrization, and tumors are examples of conditions requiring surgical intervention $(4,5)$. Permanent tracheostomy in horses can be performed in a standing position with sedation or dorsal recumbency with general anesthesia. Briefly, the cranial parts of the 2 nd to 5 th tracheal cartilages are incised including the associated skin, muscle, and tracheal cartilages. The external mucosa and skin may then be sutured with a simple interrupted method using size 2-0 vicryl $(1,5)$. One year postoperatively, the survival rate of tracheostomized horses was $97 \%$

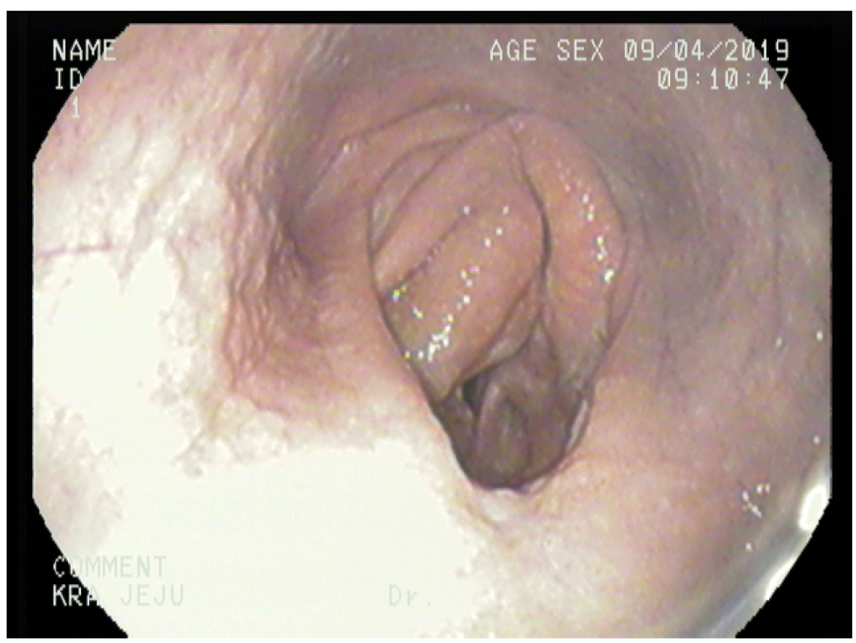

Fig. 1. Endoscopic examination of the larynx. Right-sided arytenoid chondritis and dorsal displacement of the soft palate were diagnosed with the endoscope.
(4) and $91 \%$ of the owners were satisfied with the surgery result $(1,9)$.

The purpose of this case report is to describe the clinical findings, diagnosis, treatment options, efficacy, and complications of the permanent tracheostomy in a horse with diseases causing upper respiratory tract obstruction.
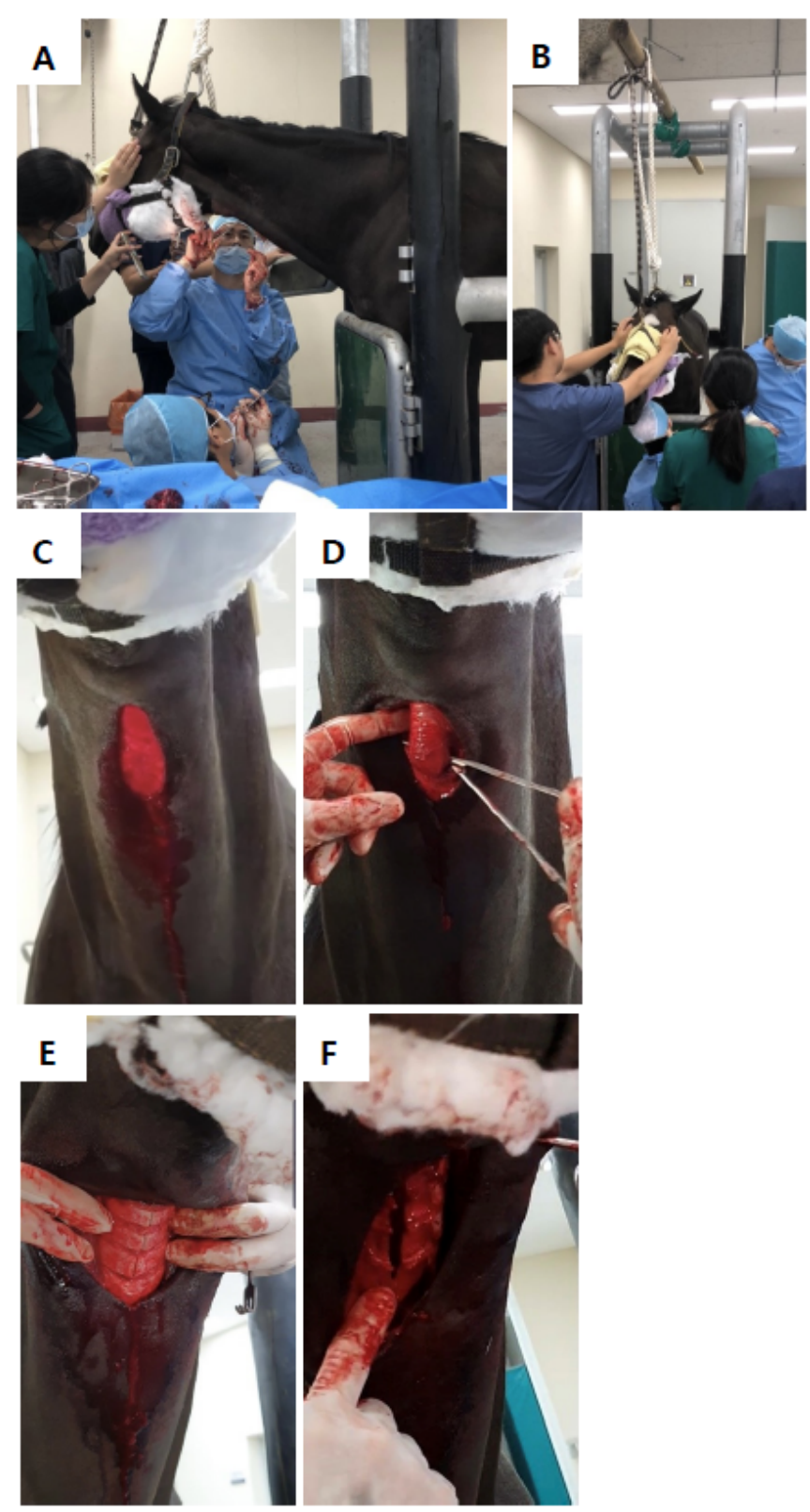

Fig. 2. The horse was restrained in a stock, and the head was tied to the roof to allow extension (A, B). A permanent tracheostomy was performed. The skin was incised (C), and the paired thyrohyoid muscles were separated and dissected (D). The tracheal cartilage was exposed (E), and the midline of the 2 nd to 5 th cartilages was resected (F). 


\section{Case}

A 10-year-old, $495 \mathrm{~kg}$ Thoroughbred mare was referred to the Jeju National University Equine Hospital with clinical signs including roaring, dyspnea, and weight loss (Body conditioning score $[\mathrm{BCS}], 3 / 9$ ). Previous medical treatment with antibiotics and non-steroidal anti-inflammatory drugs (NSAID) had failed to resolve the horse's clinical signs. There was no specific abnormality observed on radiographic examination or blood test results. On endoscopic examination, the right arytenoid cartilage was observed to be swollen asymmetrically, with the soft palate displaced dorsally; thus, the horse was diagnosed as right-sided arytenoid chondritis with concurrent dorsal displacement of the soft palate (DDSP) (Fig. 1).

Permanent tracheostomy was performed in a standing position as described previously $(1,4,5,8)$. The horse was restraint in a stock and, for extension, the head was tied to the roof (Fig. 2A, B). The horse was given $10 \mu \mathrm{g} / \mathrm{kg}$ of detomidine (Provet Detomidin, JSK Co., Ltd., Gyeonggi-do, Korea) combined with $10 \mu \mathrm{g} / \mathrm{kg}$ of butorphanol tartrate (Butorphan, Myungmoon, Seoul, Korea) intravenously and locally anesthetized with $30 \mathrm{~mL}$ of $2 \%$ lidocaine $\mathrm{HCl}$ (Lidocaine, Daihan, Korea) subcutaneously at the area to be operated, extending from the 2 nd to 5 th tracheal cartilages. The skin and subcutaneous tissue were incised over a surgical area from the 1st to 6th tracheal cartilages (Fig. 2C). Paired sternothyrohyoideus muscles covering the surgical site of the trachea were separated at the midline. Both ends of the separated muscle were grasped with forceps and each end was dissected (Fig. 2D). One-third of the cranial part of the 2nd to 5th tracheal cartilage was resected with a 20 blade, but the tracheal mucosa was maintained (Fig. 2E). After resecting the cartilage, the mucosa was cut along the midline and opened. The external mucosa and skin were sutured using a simple interrupted method with size 2-0 vicryl (polyglactin 910, Ethicon, Belgium) (Fig. 2F). The proximal and distal end and both midpoints of the stoma were sutured ahead to provide less tension on the remaining portions of the skin and mucosa. Those parts of the external skin and mucosa were sutured afterwards (Fig. 3A).

The antibiotics, penicillin $\mathrm{G}$ and dihydrostreptomycin 0.05 $\mathrm{mL} / \mathrm{kg}$ intramuscularly (PPS, Daesung, Seoul, Korea) and gentamycin $6.6 \mathrm{mg} / \mathrm{kg}$ intravenously (Samwoo Gentamycin, Samwoo median, Seoul, Korea) and the NSAID phenylbu-
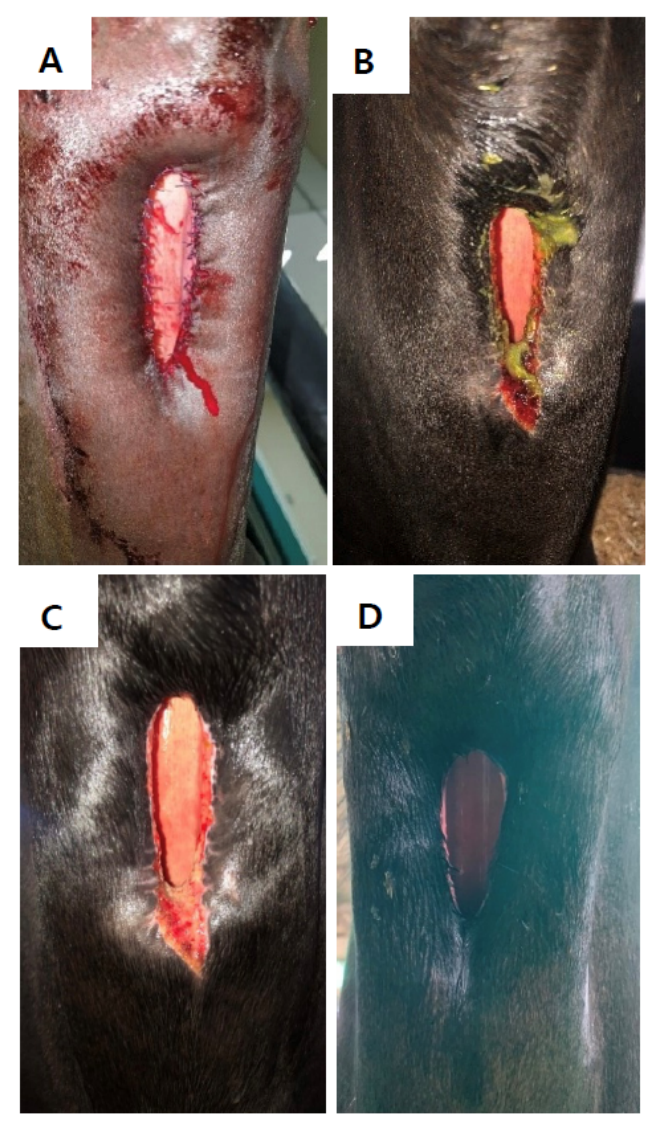

Fig. 3. Prognosis. Day 0; right after the surgery (A). Day 16; mild aspiration was observed (B). Day 22; the horse was discharged (C). Five months after surgery; the surgical site was cleansed once every 2 or 3 days (D).
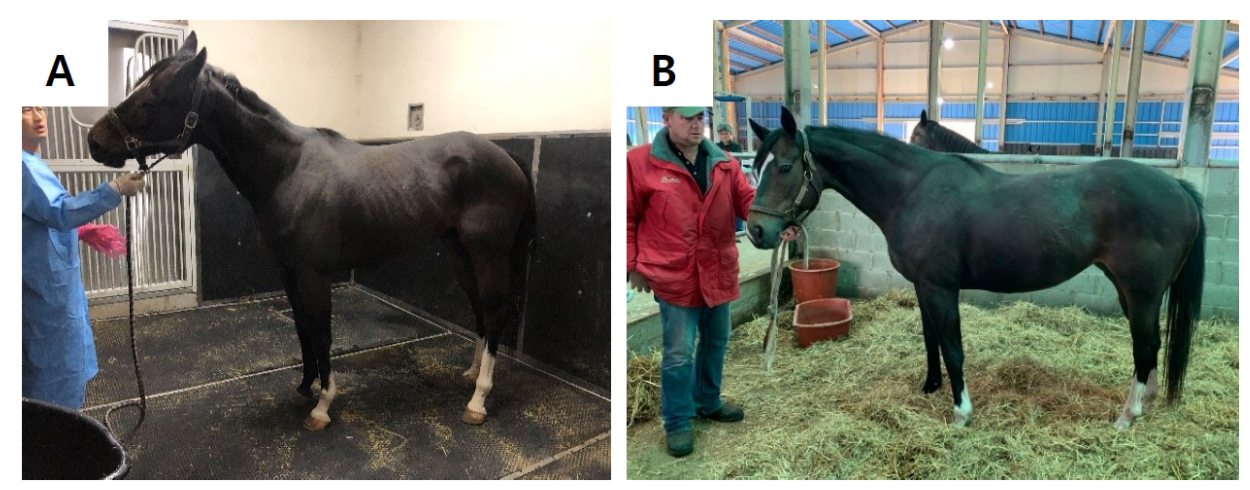

Fig. 4. The horse lost weight before surgery (body conditioning score [BCS] 3) and was discharged on day 22 (A). The horse's body weight was recovered at 4 months after the surgery (BCS 5) (B). 
tazone $10 \mathrm{mg} / \mathrm{kg}$ intravenously (Arthridine, Virbec, Seoul, Korea) were administered once a day for 10 days. The surgical site was cleansed three times a day using normal saline while the horse was hospitalized. Wet hay was provided during the early hospitalization period to prevent aspiration pneumonia, and hay in a hay net and grains was introduced gradually. Nevertheless, the food migrated to the trachea and came out of the stoma, a result suspected to be due to DDSP presence (Fig. 3B) (3). Suture dehiscence on a quarter of the lower part of the stoma, due to scratching at day 10 , was observed, the area was resutured with a size 2-0 vicryl in a simple interrupted method. The resutured area became inflamed, swollen, heated, and dehiscent. Therefore, about 5 $\mathrm{cm}$ of the lower part of the stoma was incised for drainage, and the horse was treated with antibiotics an additional 7 more days. The suture was removed on postoperative day 18, and the horse discharged on postoperative day 22 (Fig. 3C). Follow-up showed that the horse's body weight had recovered (BCS 5/9) at 4 months after the surgery, but a small amount of ingesta still trickled out of the stoma (Fig. 4). The owner cleaned the surgical site once every 2 or 3 days, and the complications resolved completely (Fig. 3D). The owner was satisfied with the result of the surgery, and the horse was successfully bred and got pregnant at 6 months after the surgery.

\section{Discussion}

Arytenoidectomy and tracheostomy are performed as surgical treatments of arytenoid chondritis. Arytenoidectomy is the removal of the arytenoid cartilage to widen the airway. The advantages of arytenoidectomy over tracheostomy are greater control of maintenance, decreased granulation of tissues, and better prognosis in racehorses $(5,13)$. The disadvantages are the necessity of general anesthesia, occurrence of a submucosal hematoma, and increased operation time (5). Also, arytenoidectomy is unlikely to provide an adequate airway, and the patient may suffer from aspiration in cases in which the remaining part of the arytenoid cartilage is affected $(5,13)$. Permanent tracheostomy is the resection of the anterior part of the trachea in order to provide a direct airway through the stoma. The main advantages of a permanent tracheostomy are its easier approach and lower risk of complications $(5,8)$. Considering that this horse was a breeding mare, a permanent tracheostomy was selected over arytenoidectomy due to the advantages described above.

The tracheostomy can be performed in either standing or recumbent positions. Although surgery in recumbency provides safety for surgeons due to better restraint of the pa- tient, general anesthesia carries a higher risk when induced and recovered, and there is an increased economic burden for the owner. In addition, in cases in which a horse has a neck disorder, it is difficult to insert a tracheal tube in recumbency, and the recumbency itself may result in tissue distortion $(4,5)$. Thus, standing surgery is more cost-effective and safer compared to recumbent surgery. Also, tissue distortion is less likely to occur in standing surgery, although standing surgery requires a greater degree of the horse's cooperation $(4,5)$. In the present case, considering that the horse had a severe obstruction in the upper respiratory tract, resulting in dyspnea, that increased the risk of side effects resulting from general anesthesia, and because the horse was relatively cooperative during the treatments, the standing surgery approach was selected.

Tracheostomy can be operated permanently or temporarily. In temporary tracheostomy, silicone tubes are inserted into the stoma temporarily, and the tubes can be removed once the cause of the upper airway obstruction is resolved $(1,5,7)$. On the other hand, in permanent tracheostomy, the stoma is formed by permanently resecting parts of the tracheal cartilages (12). Although temporary tracheostomy with medical treatment is recommended for the treatment of arytenoid chondritis $(7,10)$, in this case, the horse had not responded to previous medical treatment; thus, a permanent tracheostomy was selected, and the result met our expectations.

Surgical site infection, dehiscence, tracheal collapse, subglottic stenosis, constriction, and tissue granulation at a tracheal stoma can occur, but the incidence of such complications is low $(4,8)$. In this case, dehiscence and infection of the surgical site occurred 10 days after surgery due to scratching. The dehiscent area was resutured with the patient in a standing position. Next, the lower part of the stoma was incised to drain the pus, and the stoma border was resutured. Antibiotics and an NSAID were given, and the surgical site was cleaned daily. With the gradual introduction of food, ingesta trickled through the stoma (Fig. 3B). To promote the second healing of the resutured surgical site, antibiotics, an NSAID, and dressings were provided. Based on the complications observed in the present case, extended examinations of the horse's condition, including aspiration status, are recommended before surgery. In addition, scratching or rubbing of the surgical site after surgery should be closely monitored to prevent related complications.

It is reported that, on average, $89 \%$ of horses return to their previous use (4). Complications, such as partial dehiscence of the surgical site occur in 10\% of reported cases, while transient fever occurs in $12 \%$ and, excessive swelling in $16 \%$ of cases $(4,8)$. It is also known that $98 \%$ of horse 
owners were satisfied with the surgical outcomes and would recommend permanent tracheostomy in similar situations $(4,9)$. The average hospitalization time was reported to be 6 days with a range of 1 to 40 days (4). In this case, the horse was hospitalized for 22 days which was longer than average due to unexpected complications. In this case, the horse was discharged and went back to its intended use successfully. Mild aspiration was observed consistently, so the owner kept cleansing the stoma once every 2 or 3 days with a towel soaked in tab water or saline solution. The BCS improved from preoperative $3 / 9$ to postoperative $5 / 9$ as dyspnea improved and appetite recovered (Fig. 4). Follow-up examination showed that the horse had successfully bred and got pregnant at 6 months after the surgery. The owner was satisfied with the overall outcome of the surgery.

\section{Conclusions}

Permanent tracheostomy was performed in a 10-year-old Thoroughbred mare diagnosed with arytenoid chondritis. Even though the horse had some postoperative complications, such as aspiration and surgical site infection, the surgery was successful, and the horse fully recovered and returned to its intended use, reproductive mare, successfully. Permanent tracheostomy is thought to be efficacious in horses suffering from upper respiratory tract obstructions.

\section{Conflicts of Interest}

The authors have no conflicting interests.

\section{References}

1. Auer JA, Stick JA, Kummerle JM, Prange T. Equine surgery. St. Louis: Elsevier. 2019: 578, 611-912

2. Bolfa P, Cercone M, Dennis MM, Conan A, Grevemeyer B, Ducharme NG. Clinical and pathological features in horses with ad- vanced arytenoid chondritis. Vet Pathol 2021; 58: 91-102.

3. Carmalt JL, Johanssen B, Waldner C. Comparisons between staphylectomy and tie-forward procedures in combination with a sternothyroideus myotenectomy for the treatment of intermittent dorsal displacement of the soft palate: an observational study. Vet Surg 2016; 45: 816-823

4. Chesen AB, Rakestraw PC. Indications for and short- and longterm outcome of permanent tracheostomy performed in standing horses: 82 cases (1995-2005). J Am Vet Med Assoc 2008; 232: $1352-1356$.

5. Hawkins J. Advances in equine upper respiratory surgery. Ames: Wiley Blackwell. 2015: 87-135, 231-235, 272-275.

6. Hughes KJ, McGorum BC, Love S, Dixon PM. Bilateral laryngeal paralysis associated with hepatic dysfunction and hepatic encephalopathy in six ponies and four horses. Vet Rec 2009; 164 : 142-147.

7. Mazzaferro EM. Temporary tracheostomy. Top Companion Anim Med 2013; 28: 74-78.

8. McClure SR, Taylor TS, Honnas CM, Schumacher J, Chaffin MK, Hoffman AG. Permanent tracheostomy in standing horses: technique and results. Vet Surg 1995; 24: 231-234.

9. Rakestraw PC, Eastman TG, Taylor TS, Schumacher J, Wright L. Long-term outcome of horses undergoing permanent tracheostomy: 42 cases. Proc Am Assoc Equine Pract 2000; 46: 111-112.

10. Saulez MN, Slovis NM, Louden AT. Tracheal perforation managed by temporary tracheostomy in a horse. J S Afr Vet Assoc 2005; 76: $113-115$

11. Stick JA. Instrumentation and techniques in respiratory surgery Vet Clin North Am Equine Pract 1996; 12: 351-372.

12. Suzuki M, Tsunoda A, Shirakura S, Sumi T, Nishijima W, Kishimoto S. A novel permanent tracheostomy technique for prevention of stomal stenosis (triangular tracheostomy). Auris Nasus Larynx 2010; 37: 465-468.

13. Tulleners EP, Harrison IW, Mann P, Raker CW. Partial arytenoidectomy in the horse with and without mucosal closure. Vet Surg 1988; 17: 252-257. 